\title{
Navigated versus Non-Navigated Intraoperative Ultrasound: Is There Any Impact on the Extent of Resection of High-Grade Gliomas? A Retrospective Clinical Analysis
}

\author{
Mirjam Renovanz ${ }^{1,2}$ Anne-Katrin Hickmann ${ }^{2}$ Christine Henkel ${ }^{2}$ Minou Nadji-Ohl ${ }^{2}$ Nikolai J. Hopf ${ }^{2}$ \\ ${ }^{1}$ Department of Neurochirurgie, Universitätsmedizin Mainz, Mainz, \\ Germany \\ 2 Department of Neurochirurgie, Klinikum Stuttgart, Stuttgart, \\ Germany \\ Address for correspondence Mirjam Renovanz, MD, Department of \\ Neurosurgery, University Mayence, Langenbeckstraße 1, 55131 \\ Mayence, Germany (e-mail: mirjam.renovanz@unimedizin-mainz.de).
}

J Neurol Surg A 2014;75:224-230.

\begin{abstract}
Keywords

- glioma

- ultrasound

- extent of resection

- navigation

Introduction The extent of tumor resection is a significant predictor of survival in highgrade gliomas. In recent years, several authors showed the benefit of intraoperative ultrasound partially matched with magnetic resonance imaging (MRI). The aim of this study was to find out if intraoperative neuronavigation in combination with intraoperative ultrasound has any impact on the complete resection of gliomas. A comparison between the ultrasound-controlled resection of brain tumors and operations controlled by navigated ultrasound was performed.

Materials and Methods A total of 92 patients (54 men and 39 women) with a mean age of 53.2 years underwent 93 operations over a period of 4 years (2007-2010). They harbored a tumor with suspicion of glioma; 32 of them had undergone previous surgery, and additional chemotherapy, and 29 of them had undergone irradiation. Overall, 49 operations were performed with navigated ultrasound (group A) and 44 with nonnavigated ultrasound (group B). A standardized early postoperative MRI was performed . Complete or gross total resection (GTR) was defined by a resection of $\geq 95 \%$ of the tumor. Skin incision and craniotomy were planned after registration of the neuronavigation system. The ultrasound system was used systematically before and after opening the dura, and during and at the end of resection.

Results GTR could be achieved in 28 of 49 cases in group A and in 23 of 44 cases in group B. In group A, sensitivity and specificity of tumor remnants detected by ultrasound were higher than in group B. Concerning recurrent gliomas, the sensitivity of ultrasound visualizing tumor remnants was lower than in primary tumors. In case of preoperatively planned GTR, in both groups (navigated and non-navigated ultrasound) similar tumor remnant sizes were postoperatively detected by MRI. In nine cases the removal was incomplete because of eloquently located tumors. There was no significant difference between navigated and not-navigated ultrasound concerning GTR $(p>0.05)$.
\end{abstract}

received

July 19, 2012

accepted after revision

June 7, 2013

published online

March 12, 2014 (c) 2014 Georg Thieme Verlag KG Stuttgart · New York
DOI http://dx.doi.org/ $10.1055 / \mathrm{s}-0033-1356486$. ISSN 2193-6315. 
Conclusion Navigated ultrasound is an important technical tool that helps in intraoperative orientation. Further prospective investigation is needed to assess the impact on GTR.

\section{Introduction}

The extent of tumor resection is a significant predictor of survival in patients with low- and high-grade gliomas., ${ }^{1,2}$ Several technical tools are used during surgery to increase safety and to improve the clinical outcome. Nowadays neuronavigation systems and ultrasound are almost ubiquitously available. In recent years, several authors were able to show the benefit of two-dimensional (2D) and three-dimensional (3D) intraoperative ultrasound partially matched with magnetic resonance imaging (MRI). ${ }^{3-7}$ For example, neuronavigation allows the neurosurgeon to plan the optimal approach, to perform a craniotomy more precisely, and to avoid damaging healthy brain tissue. It is also helpful for intraoperative orientation. ${ }^{8,9}$ Ultrasound facilitates real-time intraoperative imaging because the loss of cerebrospinal fluid (CSF) and surgical manipulation may alter the position of the tumor and cause brain shift. It is useful in the localization of tumors, in the evaluation of the extent of resection (EOR), and in the identification of vessels. ${ }^{10}$ However, acquisition and interpretation of ultrasound images is user dependent and subjective, and some experience is required to obtain the preoperatively planned EOR. Therefore, linking the ultrasound probe to a neuronavigation system gives further information about the anatomical structures and could lead to an improved resection.

The aim of this retrospective study was to find out if the use of navigated ultrasound has any impact on the extent of the resection of gliomas, given the fact that the quality of images is user dependent and could be improved by neuronavigation. A comparison between ultrasound-controlled resection of high-grade gliomas and operations controlled by navigated ultrasound was performed. To our knowledge the impact of navigated ultrasound on the EOR has not been evaluated so far.

\section{Materials and Methods}

\section{Patients}

A total of 92 patients with a mean age of 53.2 years were evaluated retrospectively. They underwent 93 operations over a period of 4 years (2007-2010). The ultrasound image quality was evaluated by the two performing surgeons using a questionnaire. Fifty-four of the patients were male; 39 were female. All patients were diagnosed with a tumor suspicious of high-grade glioma: 32 of them had undergone previous surgery, and additional chemotherapy, and 24 of them had undergone irradiation. The mean follow-up was 15.9 months. Tumors $(n=9)$ located in the motor cortex, Broca or Wernicke area, thalamus, and basal ganglia were defined as eloquently localized. Therefore, in those nine cases the aim of the surgery was a subtotal resection. In two additional cases, tumor debulking/cytoreduction was planned due to the poor Karnofsky performance status (KPS) of the patients. The surgical goal for the other 82 patients was gross total resection (GTR). - Table 1 summarizes the patients' characteristics.

\section{Magnetic Resonance Imaging}

A standardized preoperative MRI (1.5-T Sonata, Siemens) was performed after a defined protocol and with special sequences (T1 contrast, MP-RAGE, T2, T2 fluid-attenuated inversion recovery [FLAIR]). In 90 of 93 cases, the tumor was contrast enhancing. In 58 of 93 preoperative MRIs, an edema could be detected.

An early postoperative MRI was performed 24 to 48 hours after surgery in all cases and compared with preoperative MRI scans. Complete or GTR ( $\geq 95 \%$ ) was defined as no solid tumor remnants in the postoperative MRI compared with the preoperative MRI scans. Additionally, a volumetric measurement of the tumor remnants in the cases of subtotal resections was performed.

Table 1 Patients' characteristics

\begin{tabular}{|l|l|}
\hline Patients' characteristics & Number (range) \\
\hline No. of operations & 93 \\
\hline Age, y, mean (range) & $53.2(24-85)$ \\
\hline Sex & $\begin{array}{l}\text { M 54 } \\
\text { F } 39\end{array}$ \\
\hline Histopathology & \\
\hline WHO grade III & 25 \\
\hline WHO grade IV & 68 \\
\hline US navigated & 49 \\
\hline US non-navigated & 44 \\
\hline Recurrence & 32 \\
WHO grade III & 8 \\
WHO grade IV & 24 \\
\hline Radiated gliomas & 24 \\
\hline WHO grade III & 3 \\
\hline WHO grade IV & 21 \\
\hline $\begin{array}{l}\text { No contrast enhancement } \\
\text { in preoperative MRI }\end{array}$ & 3 \\
\hline Tumor located in eloquent region & 9 \\
\hline
\end{tabular}

Abbreviations: US, ultrasound; WHO, World Health Organization. Note: Most of the patients harbored a glioblastoma, most of the tumors were not localized to an eloquent region, and the number of surgeries with navigated ultrasound and non-navigated ultrasound were similar. 


\section{Intraoperative Ultrasound}

Because the study is a retrospective analysis, we did not randomize the patients. Patients underwent surgery either under general anesthesia $(n=86)$ or in an asleep-awakeasleep protocol $(n=6)$. Fourteen surgeries were performed with continuous neurophysiologic monitoring (eloquently located tumors $n=9$; - Table 1). Skin incision and craniotomy were planned after registration of the neuronavigation system (Brainlab AG, Feldkirchen, Germany) was completed. A total of 49 operations were performed with navigated ultrasound (group A) and 44 with non-navigated ultrasound (group B).

-Fig. 1 shows the intraoperative situation. A Toshiba Aplio (SSA-700A Toshiba Medical Systems GmbH, Neuss, Germany) ultrasound system was used (2D, B-mode, 6.5 MHz). Using navigated ultrasound (group A), the probe was referenced to the navigation system after fixation of a pointer probe with an adapter system as shown in -Fig. 1A. In both groups, systematically before and after opening the dura, ultrasound was used to evaluate the difference of image quality with and without loss of CSF. As soon as the surgeon expected the end of the resection, he inspected the surgical field using ultrasound. If remaining tumor could be visualized, further resection was performed.

\section{Analysis and Interpretation}

Intraoperative images were interpreted as follows: "tumor," "not tumor," or "unclear," and they were documented in the report of the operation. A special questionnaire evaluating the image quality of the ultrasound was used. The two surgeons made statements about quality of images, correlation of ultrasound, and preoperative MRI in the several phases of resection. The quality of ultrasound images was defined by the following criteria (also shown in - Fig. 2): visualization of anatomical landmarks such as falx cerebri, ventricles, tentorium, quality of tumor demarcation, and presence or absence of artifacts like blood, air, or tamponade material.

\section{Histopathology}

Tumor samples were examined by and revealed 25 World Health Organization (WHO) grade III and 68 grade IV gliomas.

\section{Results}

\section{Extent of Resection}

GTR could be achieved in 28 of 49 cases in group A and in 23 of 44 cases in group B. In nine cases (group A: $n=4$; group B: $n=5$ ), the removal was incomplete because of tumor localization in eloquent areas.

Overall, 11 of the 41 tumor remnants shown by the postoperative MRI had been detected by intraoperative ultrasound imaging. The median tumor volume of all incomplete resections $(n=41)$ was $3.1 \mathrm{~cm}^{3}$ (standard deviation [SD]: $4.7 \mathrm{~cm}^{3}$; range: $0.36-11.7 \mathrm{~cm}^{3}$ ). In the subgroup of 11 cases, where the aim of the surgery was a subtotal resection, tumor remnant volume was $7.9 \mathrm{~cm}^{3}$ (SD: $6.6 \mathrm{~cm}^{3}$; range: $1.34-19.8 \mathrm{~cm}^{3}$ ).

In cases with preoperatively planned GTR but postoperative MRI showed subtotal resection with tumor remnant, similar median tumor remnant sizes were detected postoperatively in both groups: navigated versus non-navigated ultrasound (no significant difference; $t$-test; $p>0.05$; - Table 2 ).

In group $A$, the sensitivity and specificity of ultrasounddetecting tumor remnants were higher than in group $B$. Concerning recurrent gliomas, sensitivity visualizing tumor remnants was lower than in primary tumors. These results are shown in - Table 2. There was no significant difference between group A and group B concerning GTR $(p>0.05)$. Recurrent gliomas and primary gliomas in general also showed no significant difference in EOR: 57.3 versus 58.7\%.
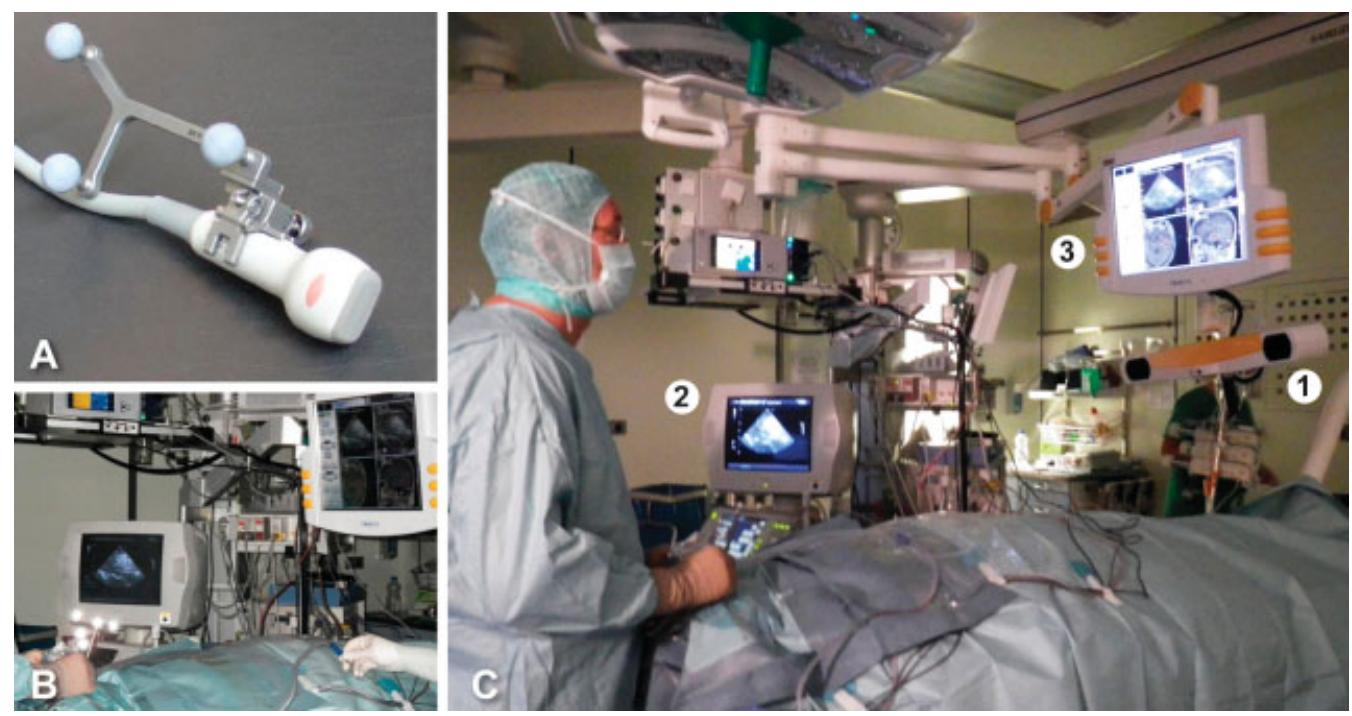

Fig. 1 (A) The ultrasound (US) probe was connected to the navigations system. (B) The probe was wrapped in a sterile foil, and the system was connected to the neuronavigation system. (C) Intraoperative situation with the camera of the neuronavigation (1), the two-dimensional US screen (2), and the neuronavigation screen (3). 


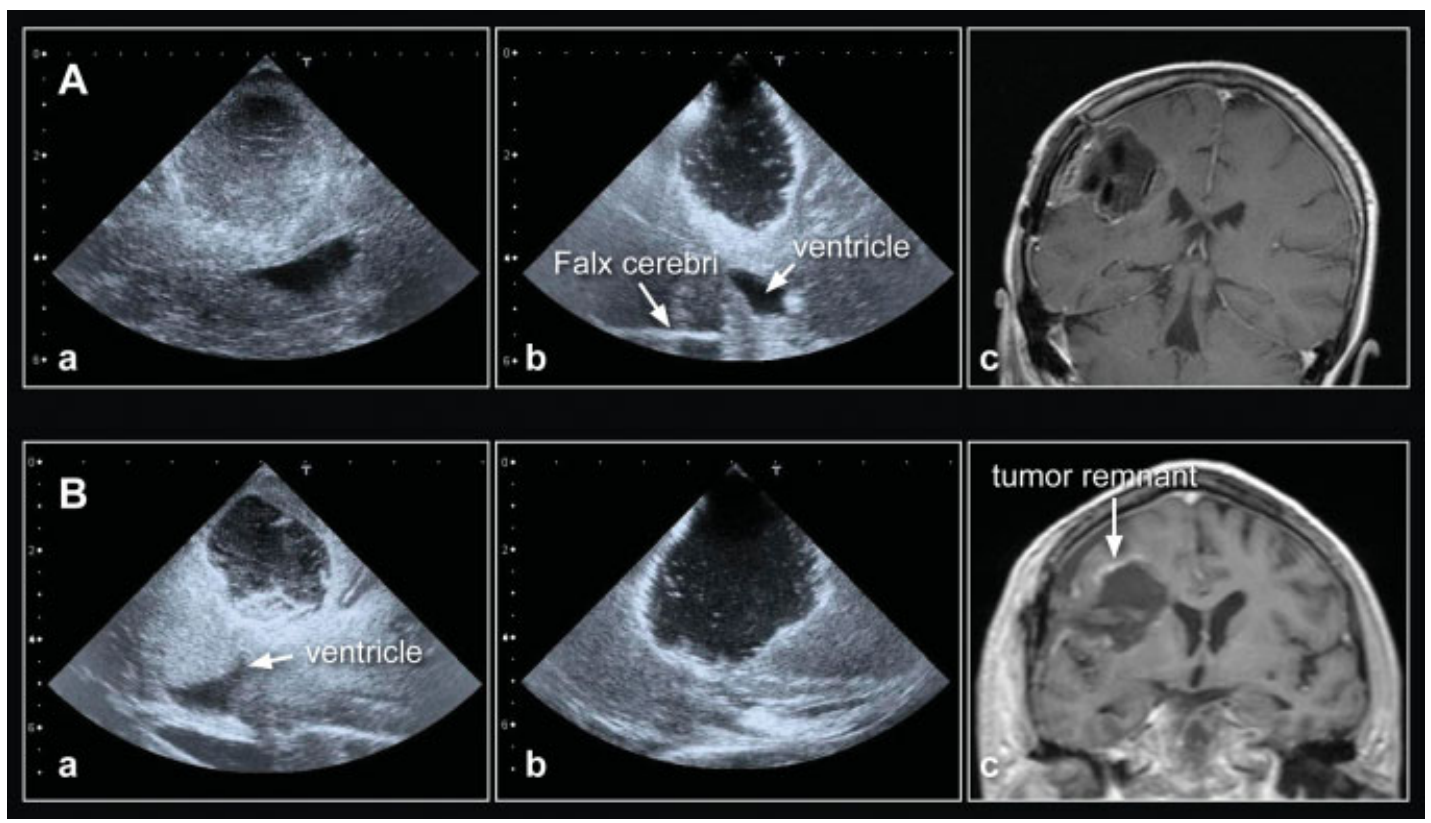

Fig. 2 (A) Example of good image quality: patient with primary glioblastoma; (a) ultrasound image, (b) image at the expected end of the resection, arrows show landmarks (Falx cerebri and ventricle) with anatomical landmarks visualized (shown in the figure), (c) postoperative magnetic resonance imaging (MRI) shows no tumor remnant. (B) Example of decreased quality of images (primary glioblastoma); (a) ultrasound, tumor, and anatomical structures are well delineated, (b) due to brain shifting the image quality has decreased at the end of the resection (arrow shows ventricle), (c) postoperative MRI shows a tumor remnant (arrow).

Table 2 Results according to navigated versus non-navigated ultrasound

\begin{tabular}{|c|c|c|}
\hline Results & $\begin{array}{l}\text { Navigated ultrasound: } \\
49 \text { cases, group A } \\
\text { (complete numbers) }\end{array}$ & $\begin{array}{l}\text { Non-navigated ultrasound: } \\
44 \text { cases, group B } \\
\text { (complete numbers) }\end{array}$ \\
\hline $\begin{array}{l}\text { GTR } \\
\text { GTR primary tumors } \\
\text { GTR recurrences }\end{array}$ & $\begin{array}{l}28(49) \\
17(32) \\
11(17)\end{array}$ & $\begin{array}{l}23(44) \\
15(29) \\
8(15)\end{array}$ \\
\hline Tumor remnant in US & 11 & 9 \\
\hline Tumor remnant in MRI & 21 & 21 \\
\hline \multicolumn{3}{|l|}{ Sensitivity of US } \\
\hline All tumors, \% & 41.7 & 19.6 \\
\hline Recurrences, \% & 12.5 & 16.7 \\
\hline \multicolumn{3}{|l|}{ Specificity of US } \\
\hline All tumors, \% & 88.7 & 82.3 \\
\hline Recurrences, \% & 88.9 & 90.0 \\
\hline $\begin{array}{l}\text { Median volume of tumor remnants } \\
\text { (SD, range) }\end{array}$ & $\begin{array}{l}1.11 \mathrm{~cm}^{3} \\
\left(0.62 \mathrm{~cm}^{3}, 0.36-2.29 \mathrm{~cm}^{3}\right)\end{array}$ & $\begin{array}{l}1.00 \mathrm{~cm}^{3} \\
\left(0.4 \mathrm{~cm}^{3}, 0.58-1.38 \mathrm{~cm}^{3}\right)\end{array}$ \\
\hline OS, mo, by a mean follow-up of 15.9 mo & $\begin{array}{l}\text { WHO'III } 27.3 \\
\text { WHO'IV } 17.1\end{array}$ & $\begin{array}{l}\text { WHO'III } 23.8 \\
\text { WHO'IV } 11.2\end{array}$ \\
\hline Mean age of patients, $y$ & 53.4 & 53.2 \\
\hline
\end{tabular}

Abbreviations: GTR, gross total resection; mo, months; MRI, magnetic resonance imaging; OS, overall survival; SD, standard deviation; US, ultrasound; WHO, World Health Organization; y, year.

Note: There was no significant difference between group A and group B concerning GTR $(p>0.05)$. Concerning primary tumors, sensitivity was higher in group A than in group B. Concerning recurrent gliomas (24 of 32 had been irradiated), sensitivity was similar in both groups. 


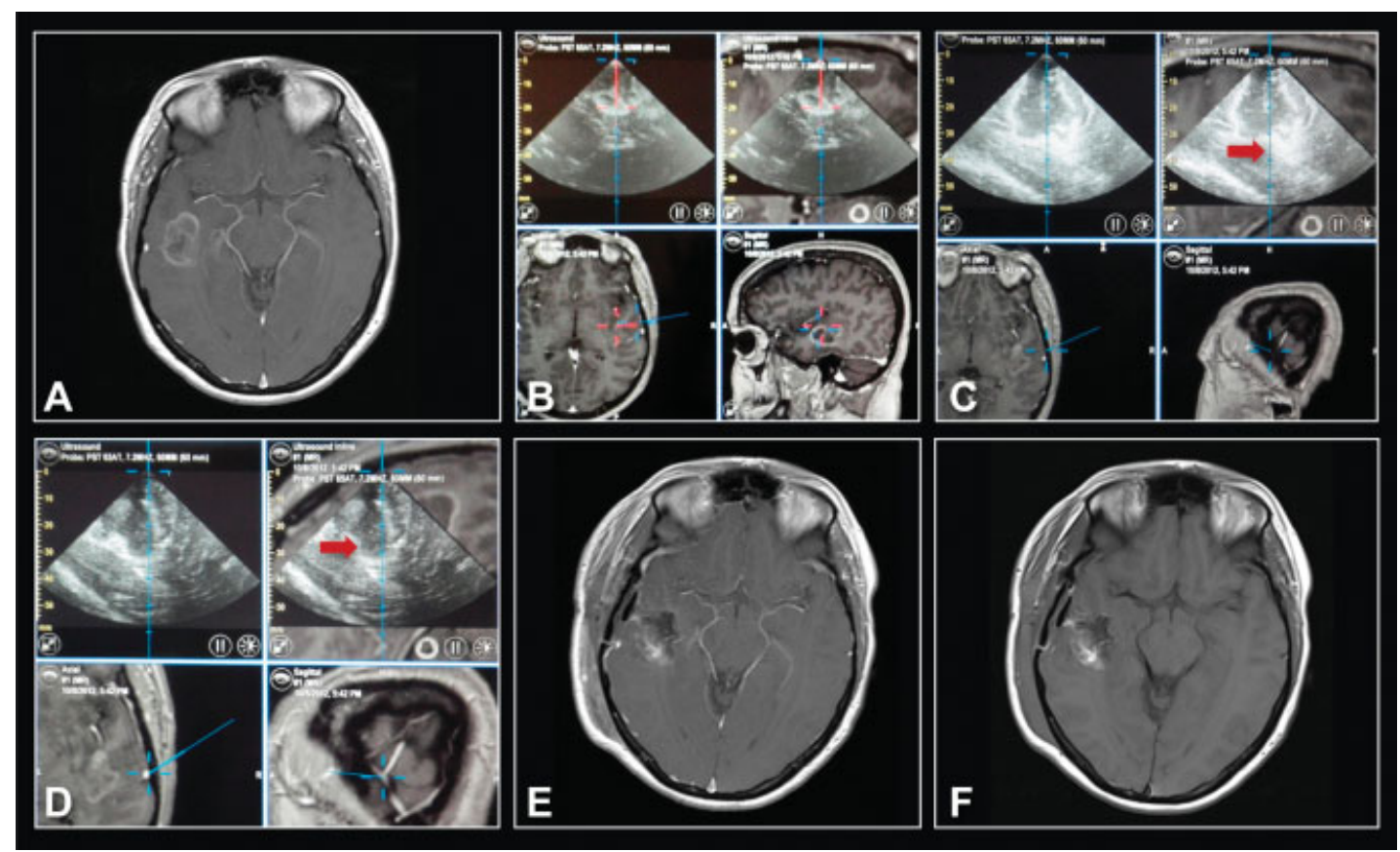

Fig. 3 Illustrating case: (A) A 41-year-old female patient, with a high-grade glioma right temporal located underwent surgery. (B) Resection was performed using navigated ultrasound, in the epidural ultrasound the tumor could be well visualized, (C, red arrow) at the expected end of resection the surgeon inspected the surgical field using ultrasound, a hyperechogenic lesion suspected of a tumor remnant could be delineated. Thus, further resection was performed. (D, red arrow) In the following ultrasound image no further tumor remnant was detected. (E, F: with and without contrast) The postoperative MRI-scan shows no tumor remnant. Histopathology revealed Glioblastoma WHO IV.

In total, 30 of the tumor remnants shown by the postoperative MRI were missed by the ultrasound. Eleven of them were recurrent gliomas, and all of them had been irradiated previously. Nine of the missed tumor remnants were located in an eloquent region, and 12 of them were $<1 \mathrm{~cm}$ in size. Only three of all tumor remnants were not contrast enhancing in the pre- and postoperative MRI, and all of them were located in an eloquent region. One of them had also been missed by ultrasound.

\section{Image Quality of Ultrasound}

On the first ultrasound image after craniotomy and before opening the dura, the tumor could be detected and localized in most of the cases. Image quality was described as "very good" or "good" in $88.6 \%$. Anatomical landmarks such as falx cerebri, ventricles, and tentorium could be visualized. After opening the dura, a second ultrasound image was performed. The results of the described image quality remained similar: In $82.3 \%$ of the cases, the surgeons described a "very good" or "good" visualization of the tumor. Only in two cases was the image quality diminished as described by the surgeons due to loss of CSF after opening the dura.

Intraoperative ultrasound images showed tumor remnants in 17 cases. Thus further removal was performed if feasible. In this phase, quality of images was judged as "good" in $69.3 \%$ because some artifacts decreased the accuracy of the images.

The overall survival (OS) (last contact with the patient or death) of the patients who underwent surgery with navigated ultrasound was in general higher than of those operated without navigated ultrasound: 18.4 months (group A) versus 13.5 months (group B). The mean age of the patients was similar in both groups: 53.4 years (group A) and 53.4 years (group B).

\section{Discussion}

\section{Factors Influencing Sensitivity of Navigated and Non- Navigated Ultrasound}

With the intention of complete resection of the solid parts of gliomas, their visualization during surgery is indispensable and poses a challenge to intraoperative imaging. On MRI the borders of malignant gliomas are defined by contrast-enhancing areas. However, the literature shows an infiltration zone existing beyond the visible tumor, so a certain intraoperative underestimation of the tumor is always to be expected. Furthermore, neuronavigation used for intraoperative orientation can cause inaccuracy because of brain shift due to tumor resection and loss of CSF.

In ultrasound, the echogenicity depends on the density of a mass and the mass difference between two adjacent tissue areas. It is also well known that the tumor borders are underestimated because gliomas are heterogeneous and mostly not well circumscribed. In addition, some artifacts like blood or air bubbles complicate the interpretation of the image. However, in general the solid part of the tumor can be visualized. The major drawback of this method is that acquisition and interpretation of the image are user dependent. ${ }^{4-6}$ Using navigated ultrasound in our study showed a trend of higher sensitivity (41.7 vs. $19.6 \%$ ) and specificity than using 
non-navigated ultrasound ( 88.7 vs. $82.3 \%$ ). Interpretation of the images is possibly more feasible with navigated ultrasound, and artifacts can be identified as such. However, concerning the EOR, no significant difference in between navigated and non-navigated could be detected.

Furthermore, previous therapies can decrease the accuracy and quality of ultrasound images because the presence of gliosis seems to increase tumor volume. ${ }^{11,12}$ In our study, almost a third of the tumor remnants missed by the ultrasound were gliomas that had been irradiated previously. Overall sensitivity for detecting tumor was lower in recurrent than in primary gliomas ( $12.5 \mathrm{vs} .41 .7 \%$ ), a phenomenon that is also described in the literature. ${ }^{13}$ Because 24 of 32 recurrent gliomas had been irradiated previously, we estimate that irradiation is an important factor influencing the image quality, sensitivity/specificity of the ultrasound, and EOR. Finally, navigation seems not to balance out this drawback in irradiated gliomas because we could not find a significant difference concerning sensitivity and specificity in the recurrent gliomas using navigated or non-navigated ultrasound (-Table 2). However, we analyzed retrospectively a limited number of operations. Further prospective data are required to obtain significant results. The number of complete resections of primary tumors and recurrences was similar (57.3 and $58.7 \%$, respectively) as also described by Gerganov et al. ${ }^{4}$ Therefore, navigation seems to lead to a better image quality but had no impact in our study on the EOR.

Furthermore, the contrast enhancement characteristics of tumors on MRI also influence the sensitivity of intraoperative ultrasound. In our group most of the tumors were contrast enhancing. Because only three of all tumor remnants were not contrast enhancing in the pre- as well as the postoperative MRIs, and one of them had been missed by the ultrasound, no meaningful analysis can be done.

\section{Sensitivity during Resection}

Additionally, we found that the sensitivity of ultrasound to detect tumor tissue decreased during resection. Comparing the first ultrasound images before and after opening the dura in most of the cases, image quality stayed stable ("good/very good image quality" in 88.6 vs. $82.3 \%$ ). Therefore, one could theorize that a second ultrasound image after opening the dura is not necessary because there was no further information benefit for the surgeons, and it takes time during the operation.

However, during resection the following factors could be identified from the operative reports leading to diminished accuracy: disadvantageous positioning of the patient and disadvantageous level of craniotomy leading to incomplete filling of the resection cavity with fluid, bleeding in the resection cavity mimicking tumor remnant, and poor differentiation of parenchyma and tumor because already edema and the bright rim effect caused hyperechogenic areas. Other authors have described these factors; this may have led to a reduced visualization of already tumor remnants. ${ }^{4,5,13-15}$ In our study we found that tumor remnants were detected by ultrasound during surgery in 17 cases (18.3\%), which led to further removal of tumor despite the fact that in this phase quality of images was judged as "good" in only $69.3 \%$.

The accuracy of neuronavigation decreased after the resection of the main part of the tumor due to brain shift, which is well investigated and described in the literature. ${ }^{15,16}$ In most of the cases, loss of CSF, brain swelling, and tumor debulking were the causal factors. The brain shift could be detected by ultrasound images but not be corrected because 2D ultrasound was used. With 3D ultrasound, a correction by intraoperative volumetric updates and fusion to preoperative MRI is theoretically possible but technically demanding. ${ }^{6,8}$

\section{Extent of Resection}

The current study demonstrates that only 11 of 41 tumor remnants could be detected by ultrasound. Smaller tumor remnants were especially not shown in the 2D ultrasound image. The volumetric assessment of tumor remnants postoperatively revealed that the median tumor remnant volume size in both groups, navigated and non-navigated ultrasound, was similar. Therefore, in our retrospective analysis, we could not find an advantage in using navigated ultrasound.

Navigated ultrasound achieved no significantly increased EOR. In the group operated with navigated ultrasound (57.1\%) and in the group operated with non-navigated ultrasound, $52.3 \%$ of the tumors could be removed completely ( $\geq 95 \%$ ), which is comparable with results of other studies in the literature. In general, EOR is an important but debated issue. EOR is associated with progression-free survival and OS of the patients. Sanai et al described in a retrospective analysis of patients with glioblastomas that significant parameters concerning OS were KPS, EOR, and postoperative tumor volume. ${ }^{2}$ They found that an EOR $>78 \%$ has a positive effect on OS. On even higher levels of EOR, this positive effect on OS continues. Stummer et al describe complete resection with $99.9 \%$ removal of the solid contrast-enhancing tumor. ${ }^{17,18}$ In our study GTR was defined by a resection of $\geq 95 \%$ of the solid tumor as also described by other authors. ${ }^{7}$

\section{Limitations of the Study}

With a mean follow-up of 16.0 months, OS was higher in the group of navigated ultrasound without reaching significant difference between the two groups. A selection bias concerning the age of the patient is not given in this case because both groups showed similar age distributions (-Table 2 ). But there is probably a certain selection bias by the surgeons because navigated ultrasound was chosen for patients with a better prognosis. A Norwegian study showed that the survival of 192 patients with glioblastoma improved after introduction of ultrasound and navigation in their department (9.6 vs. 11.9 months); adjustment for bias (main prognostic factors, e.g., age, further therapies) was performed, but interpretation still remains difficult due to the retrospective character of this evaluation. ${ }^{19}$ Another limitation of the study is that demographic parameters like KPS, social history of the patients, quality of life, and psychic factors were not evaluated. Because this is a retrospective investigation of one single center, no randomization was performed, and only two surgeons in a single center were interviewed. Both of them were used to 
ultrasound imaging techniques and probably did not profit from the better anatomical interpretation of navigated ultrasound. If younger, less experienced surgeons had been included, the results could have been more favorable toward the navigated ultrasound. Therefore, further investigations with younger surgeons should be performed. Another important question arising from this investigation is whether the combinations of intraoperative ultrasound with other tools like 5aminolevulinic acid or positron emission tomography with amino acid tracers can lead to higher extents of resection because the ultrasound image is a real-time image showing anatomical structures.

\section{Conclusion}

Navigated ultrasound is an important technical tool that aids in intraoperative orientation. To evaluate the impact for the EOR, which is influenced by multiple factors, further prospective evaluations are needed evaluating a higher number of patients in several centers.

\section{Conflict of Interest}

The authors have nothing to disclose.

\section{References}

1 Sanai N, Berger MS. Glioma extent of resection and its impact on patient outcome. Neurosurgery 2008;62(4):753-764; discussion 264-266

2 Sanai N, Polley MY, McDermott MW, Parsa AT, Berger MS. An extent of resection threshold for newly diagnosed glioblastomas. J Neurosurg 2011;115(1):3-8

3 Auer LM, van Velthoven V. Intraoperative ultrasound (US) imaging. Comparison of pathomorphological findings in US and CT. Acta Neurochir (Wien) 1990;104(3-4):84-95

4 Gerganov VM, Samii A, Akbarian A, Stieglitz L, Samii M, Fahlbusch R. Reliability of intraoperative high-resolution $2 \mathrm{D}$ ultrasound as an alternative to high-field strength MR imaging for tumor resection control: a prospective comparative study. J Neurosurg 2009;111(3):512-519

5 Unsgaard G, Gronningsaeter A, Ommedal S, Nagelhus Hernes TA. Brain operations guided by real-time two-dimensional ultrasound: new possibilities as a result of improved image quality. Neurosurgery 2002;51(2):402-411; discussion 411-412

6 Rygh OM, Selbekk T, Torp SH, Lydersen S, Hernes TA, Unsgaard G. Comparison of navigated 3D ultrasound findings with histopathology in subsequent phases of glioblastoma resection. Acta Neurochir (Wien) 2008;150(10):1033-1041; discussion 1042
7 Berntsen EM, Gulati S, Solheim O, et al. Functional magnetic resonance imaging and diffusion tensor tractography incorporated into an intraoperative 3-dimensional ultrasound-based neuronavigation system: impact on therapeutic strategies, extent of resection, and clinical outcome. Neurosurgery 2010; 67(2):251-264

8 Lindner D, Trantakis C, Renner C, et al. Application of intraoperative 3D ultrasound during navigated tumor resection. Minim Invasive Neurosurg 2006;49(4):197-202

9 Ganslandt O, Behari S, Gralla J, Fahlbusch R, Nimsky C. Neuronavigation: concept, techniques and applications. Neurol India 2002;50(3):244-255

10 Ivanov M, Wilkins S, Poeata I, Brodbelt A. Intraoperative ultrasound in neurosurgery-a practical guide. Br J Neurosurg 2010; 24(5):510-517

11 Unsgaard G, Ommedal S, Muller T, Gronningsaeter A, Nagelhus Hernes TA. Neuronavigation by intraoperative three-dimensional ultrasound: initial experience during brain tumor resection. Neurosurgery 2002;50(4):804-812; discussion 812

12 Hammoud MA, Ligon BL, elSouki R, Shi WM, Schomer DF, Sawaya R. Use of intraoperative ultrasound for localizing tumors and determining the extent of resection: a comparative study with magnetic resonance imaging. J Neurosurg 1996;84(5):737-741

13 Solheim O, Selbekk T, Jakola AS, Unsgård G. Ultrasound-guided operations in unselected high-grade gliomas-overall results, impact of image quality and patient selection. Acta Neurochir (Wien) 2010;152(11):1873-1886

14 Woydt M, Krone A, Becker G, Schmidt K, Roggendorf W, Roosen K. Correlation of intra-operative ultrasound with histopathological findings after tumor resection in supratentorial gliomas. a method to improve gross total tumor resection. Acta Neurochir (Wien) 1996;138(12):1391-1398

15 Letteboer MM, Willems PW, Viergever MA, Niessen WJ. Brain shift estimation in image-guided neurosurgery using 3-D ultrasound. IEEE Trans Biomed Eng 2005;52(2):268-276

16 Comeau RM, Sadikot AF, Fenster A, Peters TM. Intraoperative ultrasound for guidance and tissue shift correction in imageguided neurosurgery. Med Phys 2000;27(4):787-800

17 Stummer W, Tonn JC, Mehdorn HM, et al; ALA-Glioma Study GroupCounterbalancing risks and gains from extended resections in malignant glioma surgery: a supplemental analysis from the randomized 5-aminolevulinic acid glioma resection study. Clinical article. J Neurosurg 2011;114(3):613-623

18 Stummer W, Reulen HJ, Meinel T, et al; ALA-Glioma Study Group Extent of resection and survival in glioblastoma multiforme: identification of and adjustment for bias. Neurosurgery 2008;62 (3):564-576; discussion 564-576

19 Sæther CA, Torsteinsen M, Torp SH, Sundstrøm S, Unsgård G, Solheim O. Did survival improve after the implementation of intraoperative neuronavigation and 3D ultrasound in glioblastoma surgery? A retrospective analysis of 192 primary operations. J Neurol Surg A Cent Eur Neurosurg 2012;73(2): 73-78 